\title{
3D pressure imaging of an aircraft propeller blade-tip flow by phase-locked stereoscopic PIV
}

\author{
D. Ragni • B. W. van Oudheusden • \\ F. Scarano
}

Received: 1 July 2011/Revised: 6 November 2011/Accepted: 16 November 2011/Published online: 1 December 2011

(C) The Author(s) 2011. This article is published with open access at Springerlink.com

\begin{tabular}{|c|c|c|}
\hline Abstract The flow field at the tip region of a scaled DHC & 0 & Rotational frequency $(\mathrm{Hz})$ \\
\hline Beaver aircraft propeller, running at transonic speed, has & $V$ & Velocity $(\mathrm{m} / \mathrm{s})$ \\
\hline en investigated by means of a multi-plane stereoscopic & $J$ & Advance ratio (-) \\
\hline particle image velocimetry setup. Velocity fields, & $D$ & Propeller diameter (D) \\
\hline phase-locked with the blade rotational motion, are acquired & $\mathrm{V}$ & Control volume $\left(\mathrm{m}^{3}\right)$ \\
\hline across several planes perpendicular to the blade axis and & $S$ & Surface of the control volume $\left(\mathrm{m}^{2}\right)$ \\
\hline erged to form a 3D measurement volume. Transonic & $S_{\text {blade }}$ & Surface of the blade $\left(\mathrm{m}^{2}\right)$ \\
\hline nditions have been reached at the tip region, with a & $f$ \# & Lens focal length/lens aperture (-) \\
\hline volution frequency of $19,800 \mathrm{rpm}$ and a relative & $C_{P}$ & Pressure coefficient (-) \\
\hline ee-stream Mach number of 0.73 at the tip. The pressure & $\Delta \mathrm{px}$ & Free-stream pixel shift (px) \\
\hline eld and the surface pressure distribution are inferred from & $\rho$ & Density $\left(\mathrm{kg} / \mathrm{m}^{3}\right)$ \\
\hline the $3 \mathrm{D}$ velocity data through integration of the momentum & $\lambda$ & Size of the structure to be resolved (m) \\
\hline avier-Stokes equation in differential form, allowing for & $w s$ & Window size (px or $\mathrm{m})$ \\
\hline the simultaneous flow visualization and the aerodynamic & $\omega$ & Vorticity $(\mathrm{Hz})$ \\
\hline ads computation, with respect to a reference frame & $\alpha$ & Angle of attack (deg) \\
\hline oving with the blade. The 1 & $x, y, z$ & of reference $(\mathrm{m}, \mathrm{m}, \mathrm{m})$ \\
\hline further integrated by means of & $\sigma$ & ity (q.d.) \\
\hline eld the aerodynamic sectional force components as well & $\mathrm{d} x, \mathrm{~d} y, \mathrm{~d} z$ & Grid \\
\hline the blade torsional moment. A steady Reynolds averaged & $M$ & Ma \\
\hline Navier-Stokes numerical simulation of the entire propeller & $\operatorname{Re}$ & mber $(-)$ \\
\hline model has been used for comparison & $y+$ & Dimensionless wall-dista \\
\hline data. & $y_{0}$ & First \\
\hline & $\varepsilon_{d z}$ & on the spacing $\mathrm{d} z(\mathrm{~mm})$ \\
\hline mbols & $\tau$ & Shear \\
\hline Number of blades (-) & $I_{\varepsilon}$ & Peak locking uncertainty (px) \\
\hline Propeller radius $(\mathrm{m})$ & $\kappa$ & Uncertainty amplification factor (-) \\
\hline Hub radius $(\mathrm{m})$ & $T$ & Temperature $(\mathrm{K})$ \\
\hline Blade maximum chord $(\mathrm{m})$ & $u, v, w$ & Cartesian velocity decomposition $(\mathrm{m} / \mathrm{s})$ \\
\hline Radial position (m) & $\begin{array}{l}R_{a} \\
T^{\prime}\end{array}$ & $\begin{array}{l}\text { Specific gas constant }\left(\mathrm{J} \mathrm{kg}^{-1} \mathrm{~K}^{-1}\right) \\
\text { Sectional thrust }(\mathrm{N} / \mathrm{m})\end{array}$ \\
\hline & $Q^{\prime}$ & Sectional torque force $(\mathrm{N} / \mathrm{m})$ \\
\hline $\begin{array}{l}\text { Ragni }(\bowtie) \cdot \text { B. W. van Oudheusden } \cdot \text { F. Scarano } \\
\text { aculty of Aerospace Engineering, } \\
\text { Pelft University of Technology, Delft, } \\
\text { he Netherlands } \\
\text {-mail: D.Ragni@ @tudelft.nl }\end{array}$ & $\begin{array}{l}A^{\prime} \\
M_{A}^{\prime} \\
d_{T E} \\
P\end{array}$ & $\begin{array}{l}\text { Sectional axial force }(\mathrm{N} / \mathrm{m}) \\
\text { Sectional blade torsion }(\mathrm{Nm} / \mathrm{m}) \\
\text { Distance from the trailing edge }(\mathrm{m}) \\
\text { Pressure }(\mathrm{Pa})\end{array}$ \\
\hline
\end{tabular}


$N \quad$ Number of samples (-)

$\gamma \quad$ Specific heat ratio (-)

\section{Subscripts, superscripts}

$x, y, z \quad$ Along the Cartesian component

$\infty \quad$ Free-stream

$R \quad$ Relative

1 Sectional

o Total/stagnation

$T \quad$ Transport

1, 2 Different positions

*q.d Quantity dependent

\section{Introduction}

The increased demand of low fuel consumption and high efficiency has encouraged the use of aircraft propellers as propulsive devices in the aeronautical field. To be competitive with other devices flying in the high-subsonic regime, such as turbofans and turbojets, modern aircraft propellers usually need to operate at high revolution frequencies and severe blade loading, typically determining the coexistence of high Reynolds number and compressibility effects (Favier et al. 1989). Early studies in aircraft propellers reported that under these conditions, fatigue is enhanced to such an extent of being the cause of the system failure, in particular, in the presence of cyclic blade loading (Stepnov et al. 1977). The most relevant loading components are typically the aerodynamic and centrifugal forces acting on the blade, together with the vibrations produced by the inhomogeneous torque distribution, critical for piston-engines. Most of these contributions are flow dependent, therefore difficult to be quantified, due to the complexity arising from instrumenting rotating objects. Despite the safety precautions and calculations from failure mechanics, the prediction of the in-flight blade loading is still uncertain in the design phase (Kushan et al. 2007); hence the causes of the failure of the system have to be investigated "a-posteriori" by optical micrographs showing the propagation of the corrosion on the damaged surface (e.g., Cessna trainer take-off accident, Lee et al. 2004).

The prediction of the blade loading profile in the transonic regime developed from the analytical extension of the potential function to subsonic compressible flows (Ludford 1951) and from the reformulation of the lifting line theory by use of the small disturbance equations (Cook and Cole 1978; Cheng et al. 1981). With the advances of computational fluid-dynamics, the aerodynamic load prediction broadened considerably, extending the data availability up to the supersonic regime and leading toward the coupling of computational structural dynamics (CSD) and computational fluid-dynamics (CFD) (a comprehensive review was compiled by Datta et al. 2007 with specific application to helicopters). Despite the rapid growth of methodologies to model a broad range of phenomena such as 3D transonic effects and dynamic stall, the co-occurrence of high Reynolds numbers with compressibility usually represents a challenge for the accurate computation of the velocity flow field (Bousquet and Gardarein 2003).

On the experimental side, the complexity involved in measuring at transonic speed on rotating objects may explain the limited availability of propeller studies. In particular, most of the experimental investigations focus on the flow field out of the propeller frame, such as the slipstream development from a propeller on a single sting (Boyle et al. 1999), the propeller-wing interaction, (Roosenboom et al. 2010) and the investigation of wakes in free-axial flight (Favier et al. 1989; Ramasamy and Leishman 2006). In this respect, the use of nonintrusive techniques such as particle image velocimetry (PIV) or laser Doppler anemometry (LDA) extended the measurement capabilities in rotating flows, due to the low degree of interference with the moving object. Moreover, the post-processing of the flow velocity and acceleration data by use of the Navier-Stokes equations has encouraged a possible coupling with loads information in both propellers (Berton et al. 2004) and airfoil applications (van Oudheusden et al. 2006).

Previous experiments in the low-speed regime performed by van Oudheusden et al. (2006) showed the potential of the contour-approach in computing both pressure and forces (Unal et al. 1997; Noca et al. 1999) on a stationary NACA642A015 profile. The authors further adapted the technique for rotating frames, measuring the velocity field in a cross-section plane at $3 / 4$ radius of a scaled propeller model, with a relative Mach number of 0.6 (Ragni et al. 2011). Throughout this analysis, it has been confirmed that pressure and forces can be obtained either by use of a stationary frame formulation or by a moving (quasi-steady) frame.

In the present study, the pressure reconstruction methodology from PIV velocity fields and the aerodynamic load computation is finally extended to the $3 \mathrm{D}$ compressible flow dynamics at the tip region of an aircraft propeller blade. The investigation aims at showing how the volume of velocity data acquired in the transonic regime can be converted, through a 3D integration technique, in a 3D pressure field distribution, the integral of which can be decomposed to characterize the cross-sectional forces distribution along the blade span. The study particularly focuses on the analysis of the cross-sectional surface pressure and thrust (the distribution of which determines the blade bending moment), with interest in the visualization of the 3D flow features, such as the development of the trailing vortex. Results are presented at a relative Mach number of 0.73 at the blade-tip; comparison data are provided by a numerical steady Reynolds averaged Navier- 
Stokes (RANS) simulation of the entire propeller, together with the computed 3D pressure fields and load distribution.

\section{Experimental procedures}

\subsection{Wind-tunnel, propeller model and operating regimes}

PIV experiments have performed on a 1/10 scale Beaver DHC propeller model in the low-speed, closed-circuit windtunnel (LST). The LST facility has a cross-section of $1.8 \mathrm{~m}$ width and $1.2 \mathrm{~m}$ height and operates up to $120 \mathrm{~m} / \mathrm{s}$ at ambient pressure $(101.3 \mathrm{kPa})$. In a similar configuration as described in Ragni et al. (2011), the 4-bladed propeller model of $236 \mathrm{~mm}$ diameter was installed in the center of the test section and driven by a $7.5 \mathrm{hp} \mathrm{electrical} \mathrm{engine,} \mathrm{mounted}$ by a supporting sting that provides cooling to the system by means of an internal water circuit. The propeller model, the supporting sting and the engine cowling were made out of stainless steel. Two embedded angular position encoders where used in the experimental setup. A first 200 pulses per revolution encoder remotely controlled the frequency of the propeller blade, maintaining it constant within $\pm 0.3 \mathrm{~Hz}$ from the prescribed regime (less than $0.1 \%$ at $330 \mathrm{~Hz}$ ). A second one with one pulse per revolution allowed the phase synchronization of the PIV measurements with the blade position, with an uncertainty corresponding to a negligible blade position jitter. The propeller system was operated at $330 \mathrm{~Hz}$ $(19,800 \mathrm{rpm})$ together with a wind-tunnel free-stream velocity of $42.3 \mathrm{~m} / \mathrm{s}$, resulting in a relative free-stream Mach number from 0.73 (at the blade-tip). The present experimental condition simulates a flight regime at about 0.6 times the maximum aircraft speed, which is generally encountered directly after take-off procedure. Details on the propeller characteristics and on the operating regimes are presented in Table 1.

In particular, in Table 1, the $R_{H}$ is the radius of the hub holding the four blades, while the solidity ratio gives an estimation of the percentage of the spanned propeller disk effectively occupied by the propeller rotor. The advance ratio $J$ is calculated through the free-stream velocity $V_{\infty}$, the revolution frequency $v$ and the propeller diameter $D$. The real/ideal pitch ratio has been derived from evaluation of the real pitch as obtained from the free-stream velocity and the one derived the geometrical pitch of the blade, which corresponds to the length the propeller would advance in ideal conditions after a full rotation in the fluid.

\subsection{PIV measurement apparatus}

A stereoscopic PIV flow investigation has been conducted to measure the blade-tip flow by merging seven crosssectional measurement planes, obtained by means of a micro-metric traversing system. The wind-tunnel has been continuously operated during the PIV images acquisition; in particular, the values of the free-stream velocity, stagnation/free-stream pressure and temperature have been averaged during the single-plane acquisition $(\sim 1-2 \mathrm{~min})$ and the data corrected for it. However, the corrections on the free-stream velocity have been found within $0.02 \mathrm{~m} / \mathrm{s}$, while an increase in the ambient temperature of about $2^{\circ} \mathrm{C}$ was found at the end of the full seven planes acquisition. The flow is seeded with particles produced by a SAFEX Twin Fog generator with SAFEX Inside Nebelfluide (mixture of dyethelene-glycol and water, with 1 micron median diameter). The tracer particles are introduced directly downstream of the wind-tunnel test section and uniformly mixed during the recirculation. Laser light is provided by a Quantel CFR200 Nd-Yag laser with $200 \mathrm{~mJ} /$ pulse energy, illuminating the field of view through laser optics forming a laser sheet of $2 \mathrm{~mm}$ thickness (about $20 \mathrm{~cm}$ wide). Two LaVision Imager Pro $L X$ cameras with $4,872 \times 3,248$ pixels (10 bit) and two Nikon lenses of $180 \mathrm{~mm}$ focal length at $f \# 8$ have been used with the LaVision Davis 7.2 software for acquisition and post-processing. Sets of 150 images have been recorded in phaselock mode at a maximum acquisition frequency of $2.5 \mathrm{~Hz}$. Cameras and laser have been simultaneously traversed by two separate mechanisms, the relative position of which determined the actual field of view. The images have been corrected for any residual misalignment through self-

Table 1 Blade geometry and summary of propeller characteristics with operating conditions

\begin{tabular}{llll}
\hline Propeller geometry (scale 1/10) & & Operating regimes \\
\hline Number of blades $N_{B}$ & 4 & Blade revolution frequency $v$ & $330 \mathrm{~Hz}$ \\
Propeller radius $R$ & $118 \mathrm{~mm}$ & Free-stream velocity $V_{\infty}$ & $42.3 \mathrm{~m} / \mathrm{s}$ \\
Propeller hub $R_{H}$ & $19 \mathrm{~mm}$ & Rotational velocity at $r / R=0.92$ & $224 \mathrm{~m} / \mathrm{s}$ \\
Blade maximum chord $c_{\max }$ & $19.4 \mathrm{~mm}$ & Chord Reynolds number at $r / R=0.92$ & 230,000 \\
Solidity ratio $c_{\max } N_{B} /(\pi R)$ & 0.21 & Advance ratio $J=V_{\infty} /(v D)$ & 0.54 \\
Blade chord at $r / R=0.75$ & $17.9 \mathrm{~mm}$ & Relative Mach at $r / R=1$ & 0.73 \\
Pitch angle at $r / R=0.75$ & $15^{\circ}$ & Real pitch/Ideal pitch & 0.81 \\
\hline
\end{tabular}




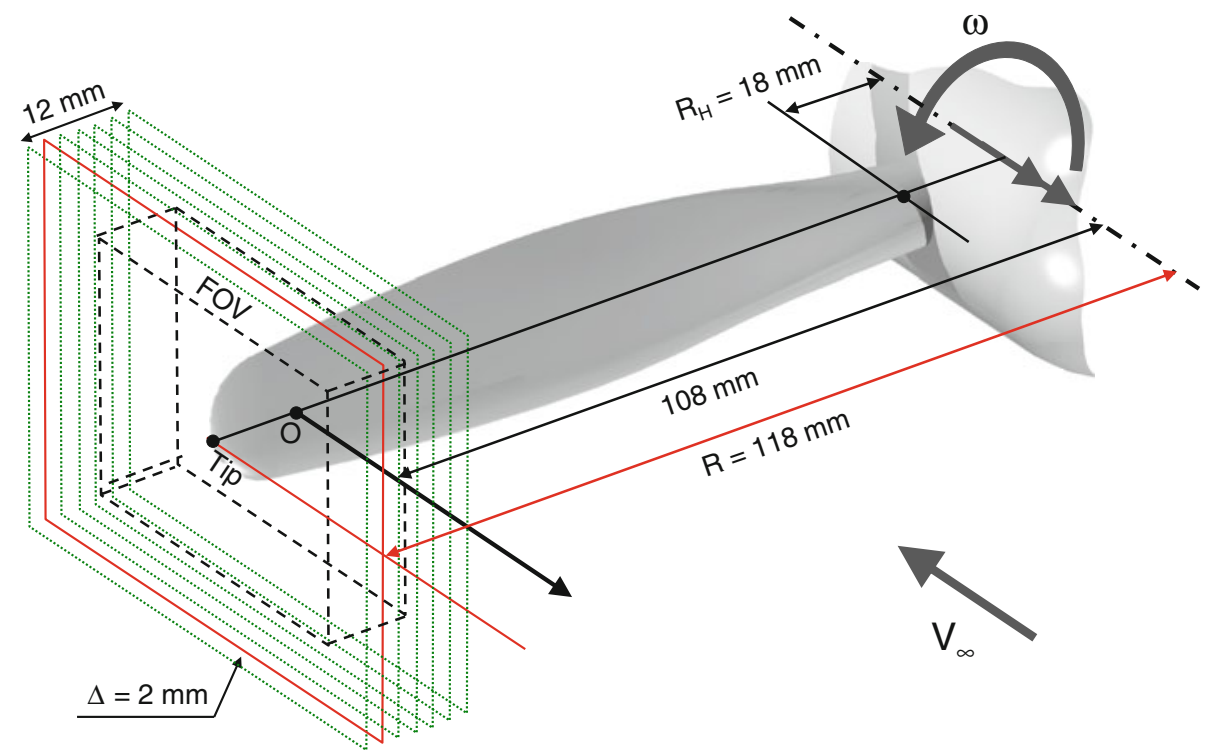

Fig. 1 Stereoscopic PIV setup and details of the apparatus

Table 2 Stereoscopic PIV setup and details of the apparatus

\begin{tabular}{llll}
\hline Imaging parameters & PIV parameters \\
\hline Camera & 2 Imager Pro LX & Software & $\begin{array}{c}\text { LaVision } \\
\text { Davis } 7.4\end{array}$ \\
Sensor format & $4,872 \times 3,248 \mathrm{px}$ & $\begin{array}{c}\text { Imaging } \\
\text { resolution }\end{array}$ & $\begin{array}{c}38-48 \mathrm{px} / \mathrm{mm} \\
\text { Window size }\end{array}$ \\
Pixel pitch & $7.40 \mu \mathrm{m}$ & $\begin{array}{c}\text { Spatial } \\
\text { resolution }\end{array}$ & $0.17-0.21 \mathrm{~mm}$ \\
Focal length & $180 \mathrm{~mm}$ & $\begin{array}{c}\text { Pulse } \\
\text { separation }\end{array}$ & $10 \mu \mathrm{s}$ \\
Magnification & 0.310 & $\begin{array}{c}\text { Free-stream } \\
\Delta \mathrm{px}\end{array}$ & $15 \mathrm{px}$ \\
Field of view & $\sim 12 \times 8 \mathrm{~cm}^{2}$ & Recordings & $150-200$ \\
FOV & & &
\end{tabular}

calibration procedure (Wieneke 2005); however, due to the accuracy of the two mechanisms, a negligible misalignment has been found. The recordings are evaluated with a window deformation iterative multi-grid (Scarano and Riethmuller 2000) with window size down to $8 \times 8$ pixels at $50 \%$ overlap $(0.20 \mathrm{~mm}$ resolution), and subsequently averaged. In Fig. 1, a schematic of the setup is presented together with a summary of the PIV parameters in Table 2 .

In order to compute the pressure field and the aerodynamic loads, the governing equations require determination of the spatial in-plane and out-of-plane velocity derivatives together with the time derivatives. However, in the present investigation, a moving frame of reference is used to formulate the governing flow equations, as already explained by the same authors in Ragni et al. (2011). Therefore, a frame of reference rotating with the blade speed is adopted to integrate the 3D pressure from the governing equations. In the absence of large unsteady effects, the quasi-steady hypothesis applies, simplifying the formulation by removing the need to evaluate the time derivative of the velocity field. In the present study, the time derivatives have been used only to confirm the equivalence of the moving frame formulation with the stationary one. The phase-delay of the system was provided by a Stanford control unit (the uncertainty of which was found to be negligible compared to the raw image scatter), allowing imaging the propeller blade at slightly shifted time instants, in particular at $\pm 5 \mu$ s from the reference. The 3D data were obtained by traversing the multiple measurement planes by a micro-meter bench in the span-wise direction of the blade, with an overall accuracy of $0.05 \mathrm{~mm}$ relative to a $\pm 2 \mathrm{~mm}$ laser sheet overall movement.

\subsection{Computational fluid-dynamic model}

To obtain a comparison test-case for the experimental study, a numerical simulation of the flow around the aircraft propeller has been prepared. The geometry of the blade has been replicated on a CAD Solid Edge model and imported in the commercial CFD simulation program Fluent V.12.1. The pitch mount angle (or pitch angle at $3 / 4$ radius) has been set to the same angle as in the experiment $\left(15^{\circ}\right.$ at $3 / 4$ propeller radius) with the experimental freestream pressure and Mach number set as boundary conditions. The full propeller has been simulated by use of a 3D steady RANS model with third-order flow discretization and Standard Wall Functions. An unstructured CFD computation of the entire propeller with $31,567,923$ cells and 


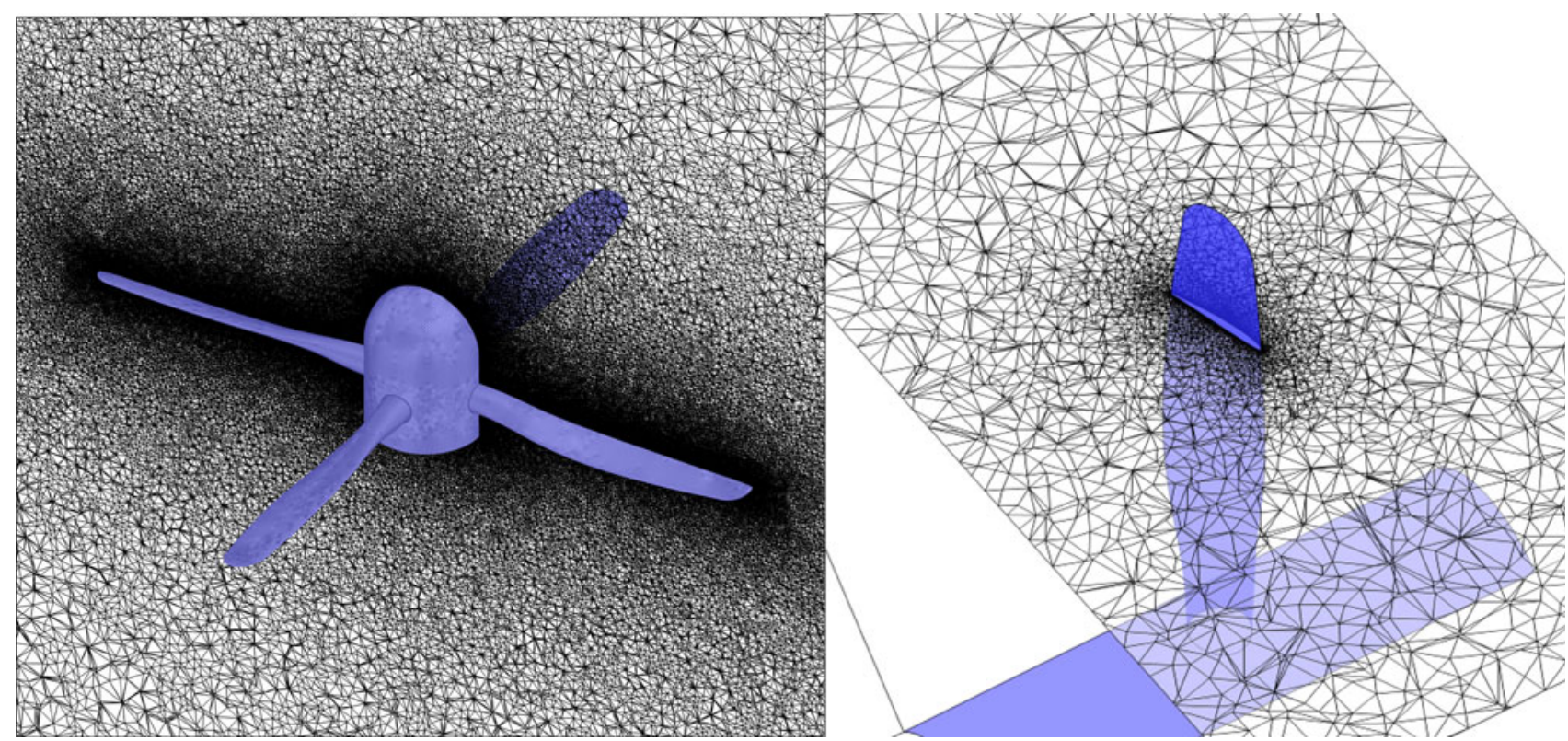

Fig. 2 Slice detail of the CFD entire propeller model (left) and of the periodical one (right)

$6,803,301$ nodes and a normal surface $y+$ of 0.8 ( $y_{0}$ at $1.1 \times 10^{-3} \mathrm{~mm}$ ) on a single steady moving frame rotating volume with a two equations $k-\varepsilon$ turbulence model was used as a PIV comparison (Fig. 2 left). The current $y^{+}$ values has been obtained through a typical boundary layer scaling of 1.2 in the normal direction, while the span-wise element size on the blade surface has been set, in the limit of the mesh generator, to $3 \%$ minimum chord.

In order to assess the influence of the turbulence scheme used, two other simulations have been set up with a periodic mesh, consisting of a $90^{\circ}$ sector meshed with three volumes, with the one of the blade on a moving frame of reference. The periodic implementation helps in optimizing the number of nodes needed, since just one of the propeller blades has to be meshed. Mapped elements on the surrounding volumes and tetrahedral in the moving one were used; the final mesh ensured a $y^{+}$value of 1 ( $y_{0}$ at $1.3 \times 10^{-3} \mathrm{~mm}$ ) in the direction normal to the blade surface with 2,069,574 nodes (Fig. 2 right).

The one equation Spalart-Allmaras and the two equations $k-\varepsilon$ turbulence model were used in the two simulations, with the same order of discretization and wall functions as in the full model; in particular, the results of the pressure coefficients on the blade surface have been compared in Fig. 3. Following the manuscript analysis, the surface pressure has been extracted on the suction side of the three planes at $r / R=91,95,98 \%$ (Fig. 3). The two turbulence models determined small differences in the pressure coefficient, generally of the order of 0.01 , except for few surface points close to the leading/trailing edge with pressure coefficient variations of 0.02 . Differences

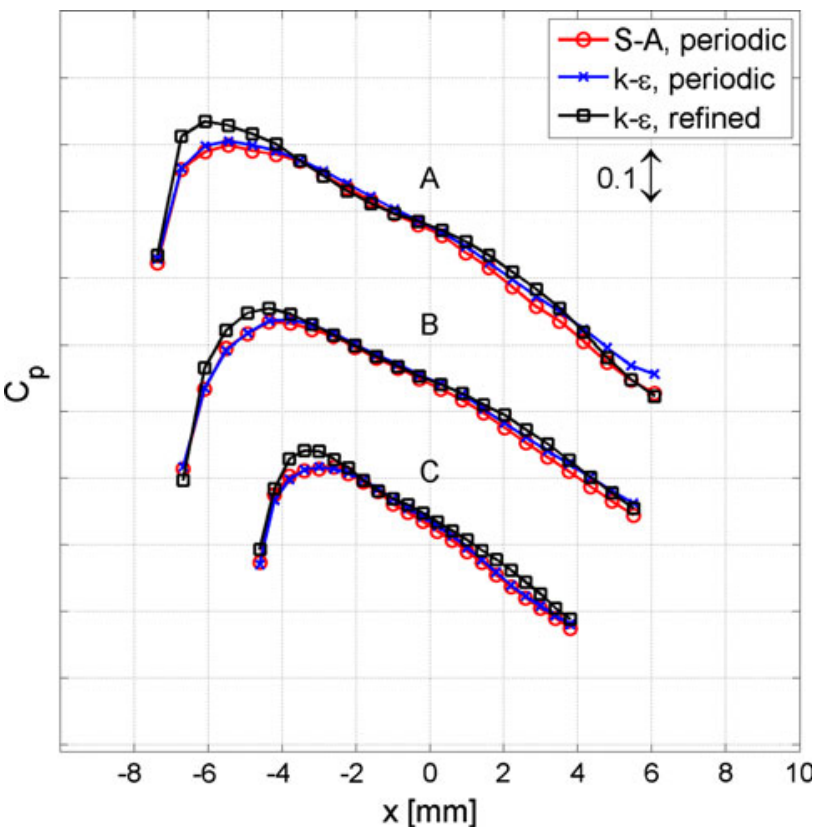

Fig. 3 Surface pressure distribution on the suction side for three planes, A: $r / R=91 \%, \mathrm{~B}: r / R=95 \%, \mathrm{C}: r / R=98 \%$, periodic mesh with Spalart-Allmaras or $\mathrm{k}-\varepsilon$ turbulence model, refined propeller mesh with $k-\varepsilon$

that are more consistent have been found between the refined full propeller model and the periodic one, due to the more accurate blade profile representation obtained through the refined computation (used throughout the manuscript). Those values range between 0.01 outside the maximum acceleration region to 0.05 compared to the periodic mesh. 


\section{Uncertainty analysis}

\subsection{PIV uncertainties}

The averaged phase-locked velocity fields are affected by random and bias error components. The first ones are primarily caused by cross-correlation uncertainty, turbulent fluctuations and phase unsteadiness resulting from jitter in the timing systems. Due to statistical convergence, the effect of these components reduces with $1 / \sqrt{ } N$ (with the number of observations $N$ ranging between 150 and 200 in the present analysis). Starting from the crosscorrelation uncertainty, a typical value of 0.1 pixel standard error is expected for a multi-pass starting window size of the order of $16 \times 16$ pixels (Westerweel 1993). On the other hand, with respect to the stationary frame of reference, the velocity fluctuations effect on the mean value is based on an root-mean-square fluctuation level of $\sigma=9.3 \mathrm{~m} / \mathrm{s}$, which is a typical value observed in the blade wake region at $r / R=92 \%$. The value corresponds to $22 \%$ of the wind-tunnel free-stream velocity and $4 \%$ of the blade speed. The minimum fluctuations observed are of the order of $\sigma=0.8 \mathrm{~m} / \mathrm{s}(1.9 \%$ of the free-stream and $0.4 \%$ of the blade speed), encountered at half propeller diameter upstream the blade leading edge. Note that this value is much higher than the actual freestream turbulence value of the LST wind-tunnel itself, which is below $0.05 \%$. The overall uncertainty on the mean velocity due to random components is assessed at $0.2 \%(0.09 \mathrm{~m} / \mathrm{s})$ of the free-stream velocity in the steady regions and $1.8 \%(0.76 \mathrm{~m} / \mathrm{s})$ in the turbulent ones. Most of the relevant sources of systematic uncertainties associated to high-speed flow effects, as discussed in the previous work by Ragni et al. (2009), have a relatively lower impact in the present PIV measurements. Both the aero-optical aberrations and particle tracers effects (Elsinga et al. 2005; Schrijer and Scarano 2007) have been found in the present investigation to be of relatively lower effect than in those encountered in the transonic airfoil study (Ragni et al. 2009), primarily due to the relatively lower acceleration values (smaller effective incidence angles), combined with the lower relaxation time of the SAFEX fog, estimated to be of the order of $1 \mu \mathrm{s}$. The uncertainty due to spatial resolution is relatively difficult to be quantified, since it depends on the location and on the ratio of the typical size $\lambda$ of the structure to be resolved and the PIV interrogation window size ws. A-posteriori evaluation on the small vortical structures encountered in the instantaneous measurements, localized in the blade wake by the Karman vortex shedding (typical vortex size $0.4-0.7 \mathrm{~mm}$ ), showed that the distribution creates a mean wake profile of $1 \mathrm{~mm}$ thickness. As shown by Schrijer and Scarano
(2008), with an in-plane PIV resolution of $0.2 \mathrm{~mm}$ (corresponding to a window size of $8 \times 8 \mathrm{px}^{2}$ ), the normalized window size $w s / \lambda$ of 0.2 can be converted into a velocity error of $<2 \%(<0.85 \mathrm{~m} / \mathrm{s})$. The error due to peak locking has been quantified by statistical analysis on the histograms of the difference between the velocity measured and its rounded-off values. The integral of the approximation error $I_{\varepsilon}$ quantifies the peak-locking velocity error to $0.03 \mathrm{px}$ corresponding to a velocity of $0.10 \mathrm{~m} / \mathrm{s}$.

\subsection{Pressure and integral loads uncertainty}

In regions of negligible vorticity, isentropic relations can be applied to retrieve pressure and pressure coefficients. Once the moving frame of reference (steady with respect to the blade) is used, it is possible to derive a direct relation between the error of the pressure coefficient, $\Delta C_{p}$, and the relative error on the (relative) velocity, $\Delta V_{R} / V_{R}$, through an error propagation parameter $\kappa$, which depends on the local flow quantities, as discussed in Ragni et al. (2011). As an example, in the present investigation, with a relative increase $V_{R} / V_{R \infty}$ of 1.2 , the resulting amplification factor is $\kappa=2.6$, hence the pressure coefficient uncertainty is in the range of $0.005-0.01$. The pressure variation in the vortical regions is obtained from integration of the 3D momentum equation, by a second-order Poisson algorithm with isentropic boundary conditions, keeping the uncertainty on $C_{p}$ of the same order as with the isentropic formulation.

In order to give an indication of the uncertainty on the sectional loads, two main inaccuracy aspects have to be quantified: an uncertainty on their localization in the $z$ direction and one on their values. Since the 3D volume has been created by merging several stereoscopic planes, the main parameters defining the uncertainty on the sectional forces localization (in case of relatively small flow gradients in the $z$ direction) are an overall misalignment $\varepsilon_{z}$ in the $z / R$ plane, and an uncertainty on the plane spacing $\varepsilon_{d z}$. In the present experiment, $\varepsilon_{z}$ is defined by the position of the laser sheet Gaussian profile, with has a relative uncertainty of $0.5 \mathrm{~mm}$ in $R=118 \mathrm{~mm}$, while $\varepsilon_{d z}$ is driven by the micro-metric bench actuator with $1 / 20 \mathrm{~mm}$ inaccuracy for $\mathrm{d} z=2 \mathrm{~mm}$. The uncertainty on the force values depends upon the combination of the previous sources of inaccuracy in the contour-approach. Therefore, the standard deviation resulting from choosing different surfaceboundary contours for the load integration for both CFD and PIV is assumed as a quantification of the experimental and computational forces uncertainties. Results are shown as error bars on the computed values in Sect. 6, while in Table 3, a summary of the most relevant sources of uncertainties are reported. 
Table 3 Summary of measurement uncertainty contributions for the velocity mean values

\begin{tabular}{llll}
\hline Baseline & Uncertainty & Reference & $\varepsilon(\mathrm{SI})$ \\
\hline Velocity & Correlation fluctuations & $\varepsilon=0.3 \mathrm{~m} / \mathrm{s}$ & $0.75 \mathrm{~m} / \mathrm{s}$ \\
& Statistical fluctuations & $\sigma_{\mathrm{u}}=9.3 \mathrm{~m} / \mathrm{s}$ & \\
& Spatial resolution & $\lambda=1 \mathrm{~mm}$ & $\leq 0.85 \mathrm{~m} / \mathrm{s}$ \\
& Peak locking & $I_{\varepsilon}=0.03 \mathrm{px}$ & $0.10 \mathrm{~m} / \mathrm{s}$ \\
Loads & Pressure coefficient & $\kappa \leq 2.8$ & 0.005 \\
& Force & & $5-15 \mathrm{~N} / \mathrm{m}$ \\
& Force localization & $\varepsilon_{\mathrm{z} 0}, \varepsilon_{\mathrm{dz}}$ & $0.5,0.05 \mathrm{~mm}$ \\
\hline
\end{tabular}

\section{Post-processing of the PIV data}

\subsection{Pressure evaluation}

The properties of the flow around the propeller blade can be evaluated either with respect to a stationary frame, with the velocity vectors as measured by an observer in the laboratory, or in a moving frame of reference, as measured from an observer moving with the angular velocity of the blade, see Ragni et al. (2011). The static fluid-dynamic quantities are independent from the flow velocity, therefore invariants in the two frames of reference. The total flow properties such as the total pressure $P_{0}$, temperature $T_{0}$ and density $\rho_{0}$, on the other hand, have different values in the two frames due to relative added energy of the moving object. Assuming no-external input of thermal energy, the flow can be considered adiabatic in the moving frame, provided to add the relative kinetic energy of the blade motion. In the absence of large unsteady effects, the flow field can also be assumed to be quasi-steady in the moving frame of reference. Therefore, in the region where the flow behaves as adiabatic and inviscid, the isentropic relations (Anderson 1991) are used to compute the pressure coefficient $C_{p}$ directly from the local relative velocity $V_{R}$ and the local Mach number $M_{R}$ :

$$
\begin{aligned}
C_{p} & =\frac{P-P_{\infty}}{\frac{1}{2} \rho_{\infty} V_{R \infty}^{2}} \\
& =\frac{2}{\gamma M_{R \infty}^{2}}\left\{\left[1+\frac{(\gamma-1)}{2} M_{R \infty}^{2}\left(1-\frac{V_{R}^{2}}{V_{R \infty}^{2}}\right)\right]^{\gamma /(\gamma-1)}-1\right\}
\end{aligned}
$$

where $\gamma$ is the heat capacity ratio of air, $\infty$ refers to the freestream quantities and ${ }_{R}$ to the ones evaluated in the moving frame. In rotational regions, if the viscous terms are neglected, the pressure can be computed with the Euler equations, which are relating the pressure gradient to the $3 \mathrm{D}$ velocity field. In the present analysis, the velocity information is obtained by combining several stereoscopic measurement planes into a 3D velocity volume. In particular, due to the quasi-steady nature of the flow in the moving frame of reference, the measurement planes have been phase-locked with the blade motion, procedure that allowed statistically characterizing the entire 3D flow, including the out-of-plane velocity components and derivatives. The pressure gradient is finally formulated in the moving frame, where the quasi-steady flow assumption simplifies the computation due to the absence of the time derivative:

$$
\frac{\bar{\nabla} P}{P}=\bar{\nabla} \ln (P)=-\frac{1}{R_{a} T}\left\{\begin{array}{l}
u_{R} \frac{\partial u_{R}}{\partial x}+v_{R} \frac{\partial u_{R}}{\partial y}+w_{R} \frac{\partial u_{R}}{\partial z} \\
u_{R} \frac{\partial v_{R}}{\partial x}+v_{R} \frac{\partial v_{R}}{\partial y}+w_{R} \frac{\partial v_{R}}{\partial z} \\
u_{R} \frac{\partial w_{R}}{\partial x}+v_{R} \frac{\partial w_{R}}{\partial y}+w_{R} \frac{\partial w_{R}}{\partial z}
\end{array}\right.
$$

As can be seen from Eq. 2, the pressure gradient is only function of the flow velocity, of the specific gas constant $R_{a}$ (for dry air assumed to be $287 \mathrm{~J} \mathrm{~kg}^{-1} \mathrm{~K}^{-1}$ ) and of the static temperature $T$, which is derived by the adiabatic assumption in the quasi-steady moving frame as in Ragni et al. (2011). In order to compute the pressure from the velocity field, Eq. 2 is rewritten as a Poisson equation, and the expression integrated through a 3D Poisson scheme. The pressure integrator being used in the present manuscript is based on the version in used by the authors in Ragni et al. (2011). The algorithm solves the Poisson equation by a inverting a linear system of equations obtained by using a second-order finite difference scheme in 3D. The algorithm was originally derived from the approach of Trefethen (2000) and applied by the authors with a variable order Spectral scheme in Ragni et al. (2010). The stencil of the code has later been simplified in a second-order finite difference scheme and rewritten in $3 \mathrm{D}$, maintaining the matrix formulation to allow for a faster reconstruction in both $2 \mathrm{D}$ and $3 \mathrm{D}$ cases. Dirichlet and Neumann boundary conditions have been applied in the present investigation in the boundary of the measurement volume, including the masked region. Dirichlet conditions obtained by imposing the isentropic pressure have been applied in the faces containing isentropic flows, while Neumann conditions have been imposed in the remaining boundaries, including the masked regions. Reynolds turbulent stresses were found to have a negligible effect in the present investigation in both the pressure reconstruction and in the momentum approach. In particular, the changes of the blade torque force with the insertion of the turbulent stresses were found in the limit of the force uncertainty, while the blade thrust remained essentially unaltered. The investigation confirmed the results in previous airfoil studies at a relatively low Reynolds number (van Oudheusden et al. 2006); 
therefore, they have not been included in the current pressure evaluation.

\subsection{Force determination by momentum integral}

The force acting on a body immersed in a fluid is the resultant of the surface pressure and shear stress distributions (Anderson 1991). However, the momentum-integral approach allows computing the force components acting on the body from their reaction on the flow by application of the integral momentum conservation in a volume $\mathrm{V}$ of surface $S$ around the body (Fig. 4), without the explicit need to evaluate the flow velocities at the surface $S_{\text {blade }}$ of the body itself.

In presence of $3 \mathrm{D}$ data, the resultant force on the object can be analyzed in terms of the span-wise distribution of the local sectional force $R^{\prime}$, sectional contribution along the $z$ axis (Fig. 4). The horizontal and vertical sectional force components $T^{\prime}, Q^{\prime}$ obtained by decomposition of the sectional resultant $R^{\prime}$ in the Cartesian $x-y-z$ frame are then computed from the following expressions:

$$
\begin{aligned}
& x,\left[\frac{N}{m}\right]: \iint_{S-S_{\text {blade }}} \rho u_{R}\left(\bar{V}_{R} \cdot \overline{d s}_{x}\right) \\
& +\frac{d}{\mathrm{~d} z} \iint_{S-S_{\text {blade }}} \rho u_{R} w_{R} \mathrm{~d} x \mathrm{~d} y=-Q^{\prime}-\iint_{S-S_{\text {blade }}}(p-\overline{\bar{\tau}}) \mathrm{d} s_{x} \\
& y,\left[\frac{N}{m}\right]: \iint_{S-S_{\text {blade }}} \rho v_{R}\left(\bar{V}_{R} \cdot \overline{d s}_{y}\right) \\
& +\frac{d}{\mathrm{~d} z} \iint_{S-S_{\text {blade }}} \rho v_{R} w_{R} \mathrm{~d} x \mathrm{~d} y=-T^{\prime}-\iint_{S-S_{\text {blade }}}(p-\overline{\bar{\tau}}) \mathrm{d} s_{y}
\end{aligned}
$$

where ${ }_{R}$ again characterizes the relative velocities components. The velocity momentum and pressure terms are the main contributors to the integral, while the stress contribution $T$, incorporating both viscous and turbulence effects, can be neglected. As can be seen from Eq. 3, the span-wise aerodynamic force $A^{\prime}$ is not considered, due to its relatively negligible contribution to the blade loading with respect to the centrifugal force in the same direction.

\section{Data analysis}

\section{$5.12 \mathrm{D}$ flow visualization}

The stereoscopic velocity fields, merged in a 3D volume of data, are visualized as different measurement planes perpendicular to the blade axis in Fig. 4. The 3D pressure as derived from integration of Eq. 2 has been projected back onto the original measurement planes, in order to be compared with the velocity information. The experimental $3 \mathrm{D}$ relative velocity and pressure contours are presented in Fig. 5 for different locations perpendicular to the blade axis, ranging from 90 to $102 \%$ of the blade radius. Due to the effect of the laser shadow, a narrow region upstream the blade is not measured. However, given the regularity of the flow in this small region, a quadratic interpolator closes the contours in the load analysis and in the data visualization (example in $I R$ region in Fig. 5), primarily where the flow is not perturbed from its free-stream value. The measurement planes identify different profile sections

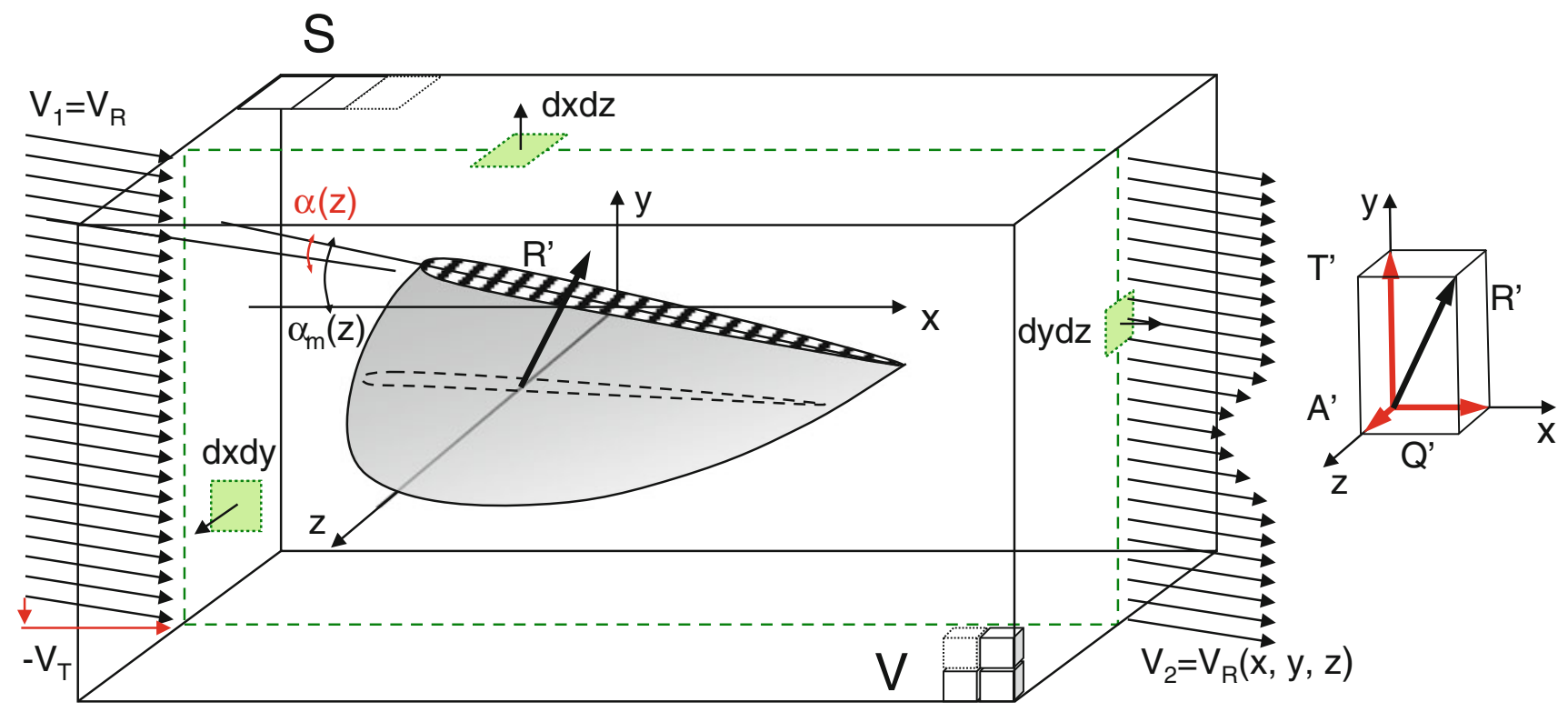

Fig. 4 Schematic of the integral momentum approach 

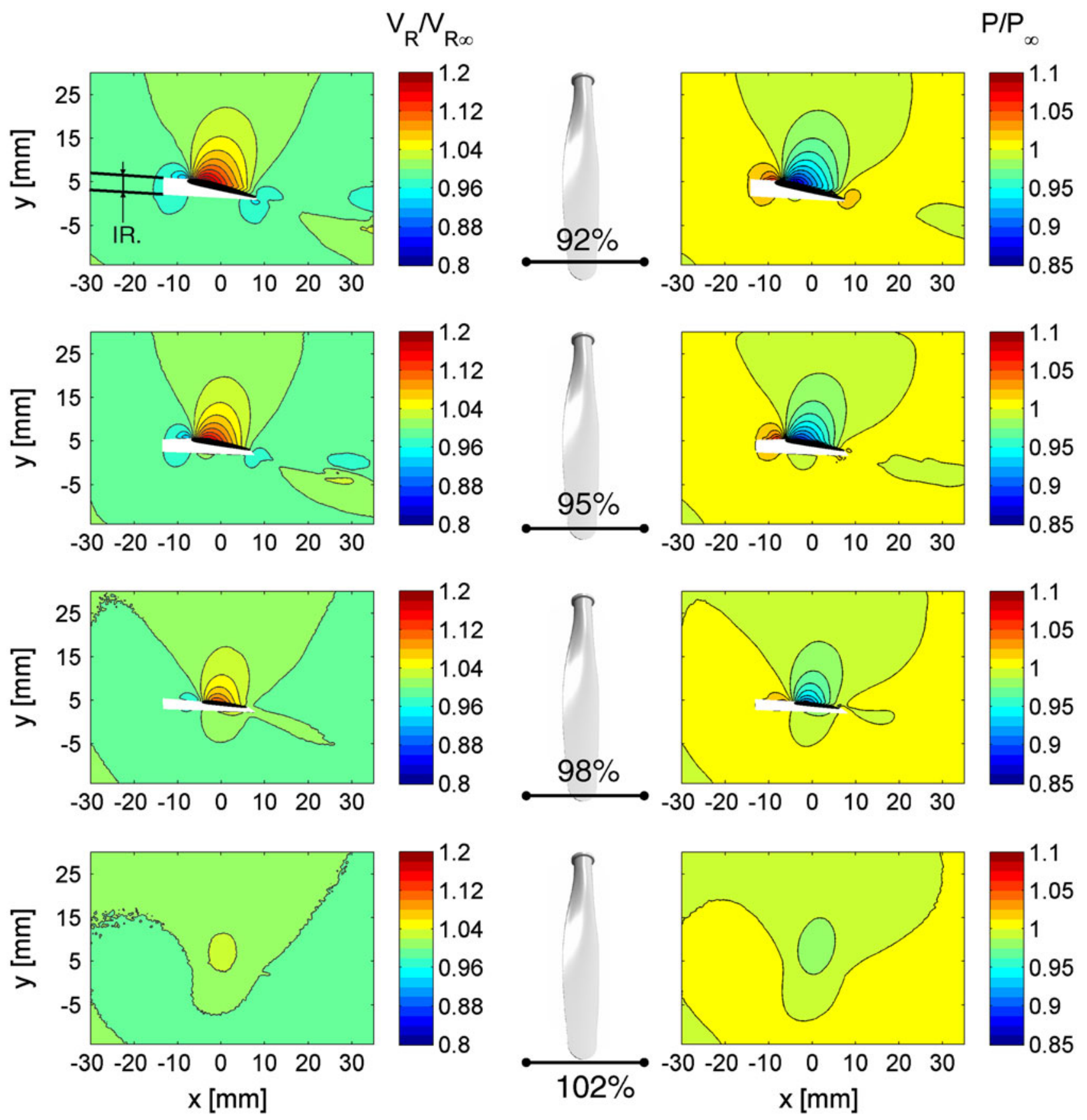

Fig. 5 Left Planar PIV visualization of the relative velocity, right PIV computed pressure from the relative velocity

along the blade shape, reflected in the evolution of the flow field along the blade span. The iso-contours of relative velocity and pressure in Fig. 4 show a net decrease in the blade angle of attack with the increase in the $z$ position, reflected in the decrease in the profile suction in the same direction from $r / R=92$ to $102 \%$. The maximum velocity variations are contained within 1.2 times the relative freestream of $223 \mathrm{~m} / \mathrm{s}$ (at $r / R=92 \%$ ), while the minimum variations are measured close to the tip of the blade $(r / R=100 \%)$, where both the relative velocity and pressure ratios are contained within 5\% of their free-stream values. As can be already seen from the present qualitative analysis, the blade has been designed to have a suction profile that extinguishes at the tip location, the only region where a negligible pressure difference across the blade profile is found. In design conditions, at a revolution frequency of $330 \mathrm{~Hz}$ (with the wind-tunnel free-stream of $42.3 \mathrm{~m} / \mathrm{s}$ ), the flow angle of attack for the nonsymmetrical profile tip at $r / R=100 \%$ is close to 1 degree. Moreover, the relatively small chord thickness 


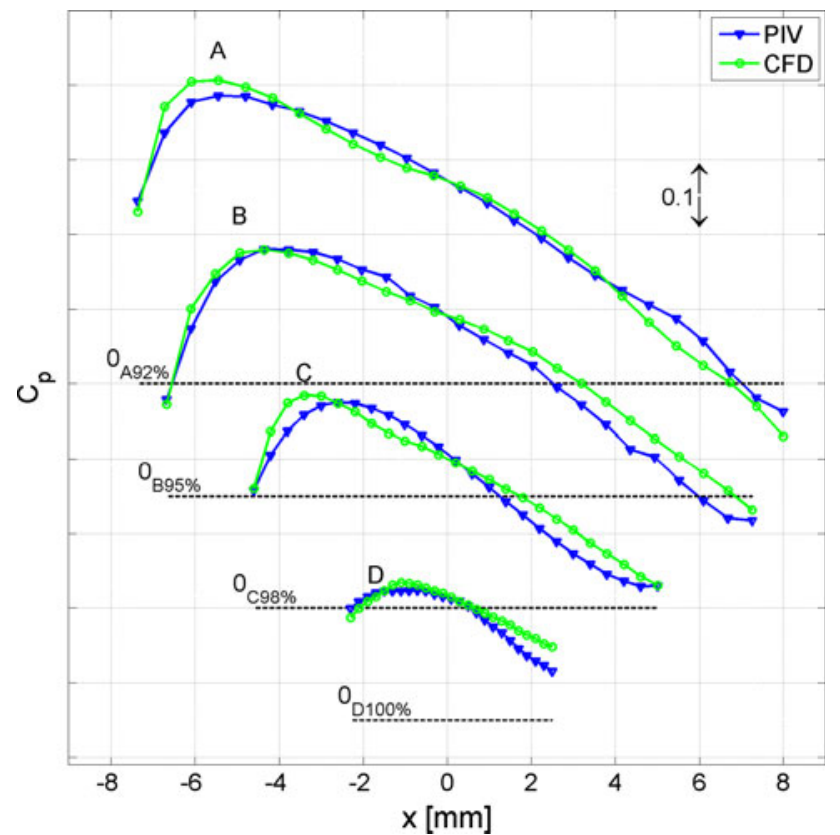

Fig. 6 Suction pressure coefficient profiles at locations $r / R=92-$ $100 \%$, comparison between PIV and CFD data

of about $1 \mathrm{~mm}$ contributes to the small extent of the pressure difference across the profile at this location. As will be discussed in the next section, the decreasing lifting distribution with increasing $z$ is still beneficial to the blade thrust generation, as it determines a relatively weaker trailing vortex, in particular reducing the pressure drag and eventually the blade torque.

\subsection{Surface pressure distribution}

The evolution of the 3D flow field around the propeller blade determines the force distribution on the model, as explained in Sect. 4.2. In particular, the surface pressure distribution represents the main contribution to the blade loading. A quantitative study of the surface pressure coefficient is carried out by the extraction of the integrated 3D pressure along different airfoil profiles, defined as intersection of the measurement planes with the blade geometry (Ragni et al. 2009). Numerical data computed from the propeller simulation as explained in Sect. 2.3 have been used as a comparison to the experimental data. In Fig. 6, the experimental surface pressure coefficient distribution has been compared to the simulated data for 4 different locations between $r / R=92 \%$ and $r / R=100 \%$. Due to the shadow region in some of the planes, the comparison has been restricted to the suction side of the propeller blade, region where the highest flow accelerations, therefore pressure variations, occur.

The pressure coefficient profiles, coherently with those presented in the previous sub-section, show a suction decrease toward the tip. Heavy separation and local sonic regions have not been encountered on the blade surface, due to the propeller operational regime close to optimum. From Fig. 6, it appears that the PIV profiles follow the numerical ones up to the highest flow accelerations, primarily identified on the $A-B$ profiles (location $r / R=92-95 \%)$. The largest discrepancies with the numerical data occur primarily on pressure recover zone of the blade profiles. In these specific regions, the inexact representation of the experimentally tested blade by the reproduced mesh determines small variations in the blade curvature, affecting the slope of the pressure coefficient curves. Typical pressure coefficient differences of the order of $\Delta C_{p}=0.02$ are appreciated in these zones, which are maintained at the blade trailing edge. At the blade-tip, the values of the pressure coefficient differences are relatively small compared to the value itself, and comparable to the measurement uncertainty of the order of 0.005-0.01. From a more careful investigation, it has been noted that the numerical data show a slightly more diffused vorticity content in the wake (see Sect. 5.3 as well), with a relatively
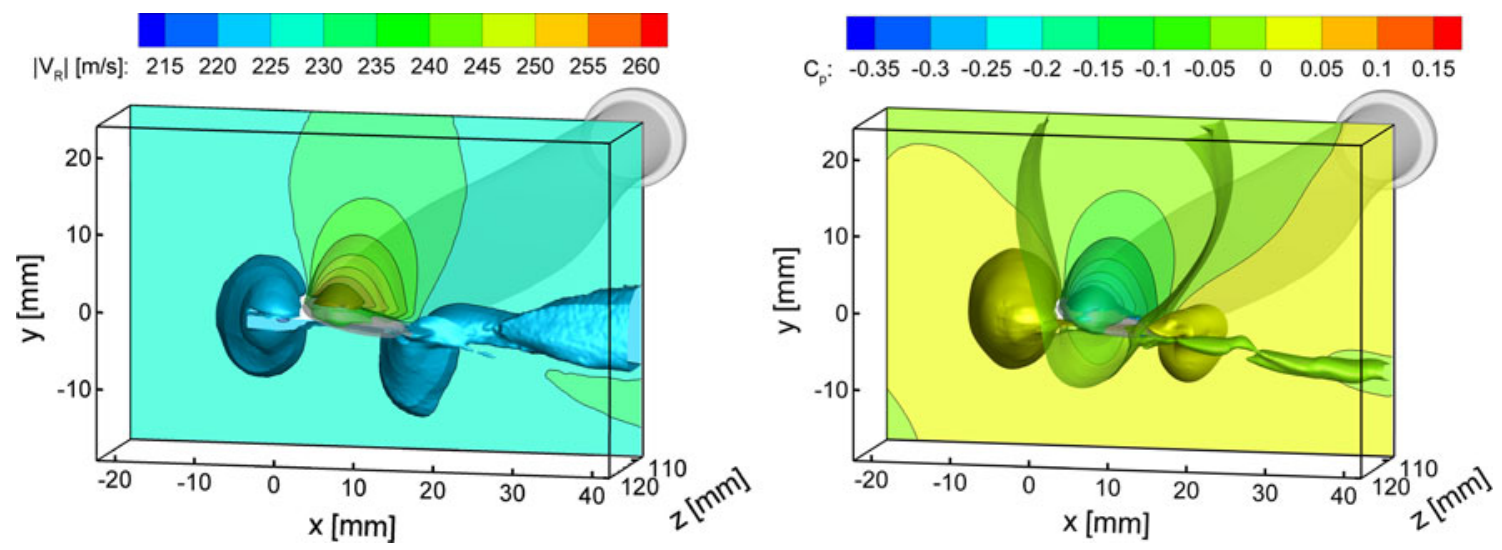

Fig. 7 3D visualization of relative velocity and pressure coefficient derived from PIV 

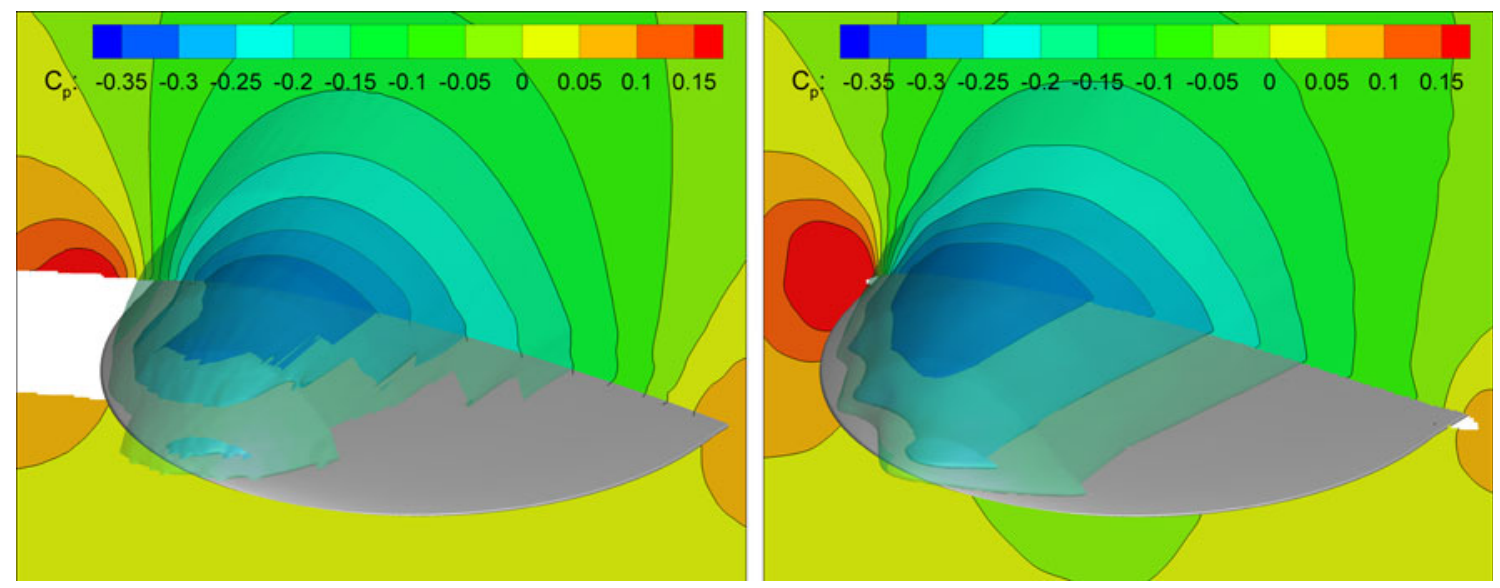

Fig. 8 Left PIV integrated pressure coefficient; right RANS computed pressure coefficient

thicker wake, determining a slower $C_{p}$ recover at the location $r / R=96-97 \%$. For the present model, investigation on the numerical data has shown that changing the turbulence model from $k-\varepsilon$ to Spalart-Allmaras causes pressure coefficient variations of $\Delta C_{p}<0.01$ in the trailing edge. On the other side, the number of elements to represent the blade surface has been found to have a stronger impact on the pressure coefficient variations, especially close to the profiles trailing edge, where the small differences on the $C_{p}$ curvature are enhanced by the approximate $\mathrm{CAD}$ representation of the real blade curvature.

\subsection{D flow visualization}

The stereoscopic velocity measurements acquired from several phase-locked planes are merged in a 3D volume and presented as 3D visualization of the flow in Fig. 7. The resulting investigated region of the propeller blade-tip extends over $60 \times 40 \times 12 \mathrm{~mm}^{3}(7 x-y$ planes with $2 \mathrm{~mm}$ spacing).

In Fig. 7, left one of the propeller blades is imaged together with the experimental 3D relative velocity field, with the relative inflow coming from the left as explained in Fig. 3. The propeller blade is mounted with a blade pitch angle $\alpha_{m}(3 / 4 R)=15^{\circ}$ in the propeller hub, with respect to the $z-x$ plane, perpendicular to the free-stream wind-tunnel velocity directed along $-y$. In the prescribed conditions, with the wind-tunnel free-stream velocity of $42.3 \mathrm{~m} / \mathrm{s}$ (see scheme in Fig. 4) and at 19,800 rpm, the aerodynamic angle of attack as computed with the tangential velocity ranges from $\alpha(r / R=92 \%) 1.5^{\circ}$ to $1.1^{\circ}$ at the tip. The contour plot and iso-surfaces in Fig. 7 show the typical features of a 3D wing moving in the flow. In particular, it can be seen how the high relative velocity on the suction side seen in Fig. 7 left corresponds to the suction peak in the pressure in Fig. 7 right, while close to the leading and trailing edge of the blade, the regions of reduction in relative velocity correspond to the pressure recovery observed in the visualization in Fig. 7 right. A qualitative analysis shows how the difference in size between the pressure recovery at the trailing edge gives a visual representation of the pressure blade drag, while the relatively lower $C_{p}$ regions starting approximately from $x=20 \mathrm{~mm}$, localizes the trailing vortex development, discussed in more details in the last part of this section. The experimental 3D surface pressure distribution is compared to the numerical one in Fig. 8. In this representation, the iso-surfaces of constant $C_{p}$ are super-imposed on the pressure coefficient contour at the volume boundary surface for both numerical and experimental data. The PIV contours (Fig. 8 left) show comparable magnitude to the numerical ones; in particular, they provide information on the extension of the maximum suction region, quantified to be about $20 \%$ of the measured blade surface and localized at about $30 \%$ of the $r / R=92 \%$ chord.

As already seen from both experimental and numerical data, the $3 \mathrm{D}$ suction distribution is reducing to 0 as the $z$ location reaches the blade radius, where the pressure jump across the blade is almost negligible. This lowpressure difference contributes to weaken the vortex formation. In this respect, the flow visualization has been extended to the blade wake, in particular, focusing on the relatively low-pressure region formation from about $x=20 \mathrm{~mm}$ (Fig. 7 right) localizing the tip vortex. The vortex visualization is carried out by use of the $3 \mathrm{D} C_{p}$ for both PIV and CFD data. Apart from a fair qualitative agreement in the flow field structure, a difference between the two results is observed as the pressure field is concerned, with a maximum experimental $C_{p}$ of -0.04 against the numerical -0.03 , the first one corresponding to about $1,300 \mathrm{~Pa}$ pressure difference with respect to the free-stream pressure. However, the minimum pressure values in the 

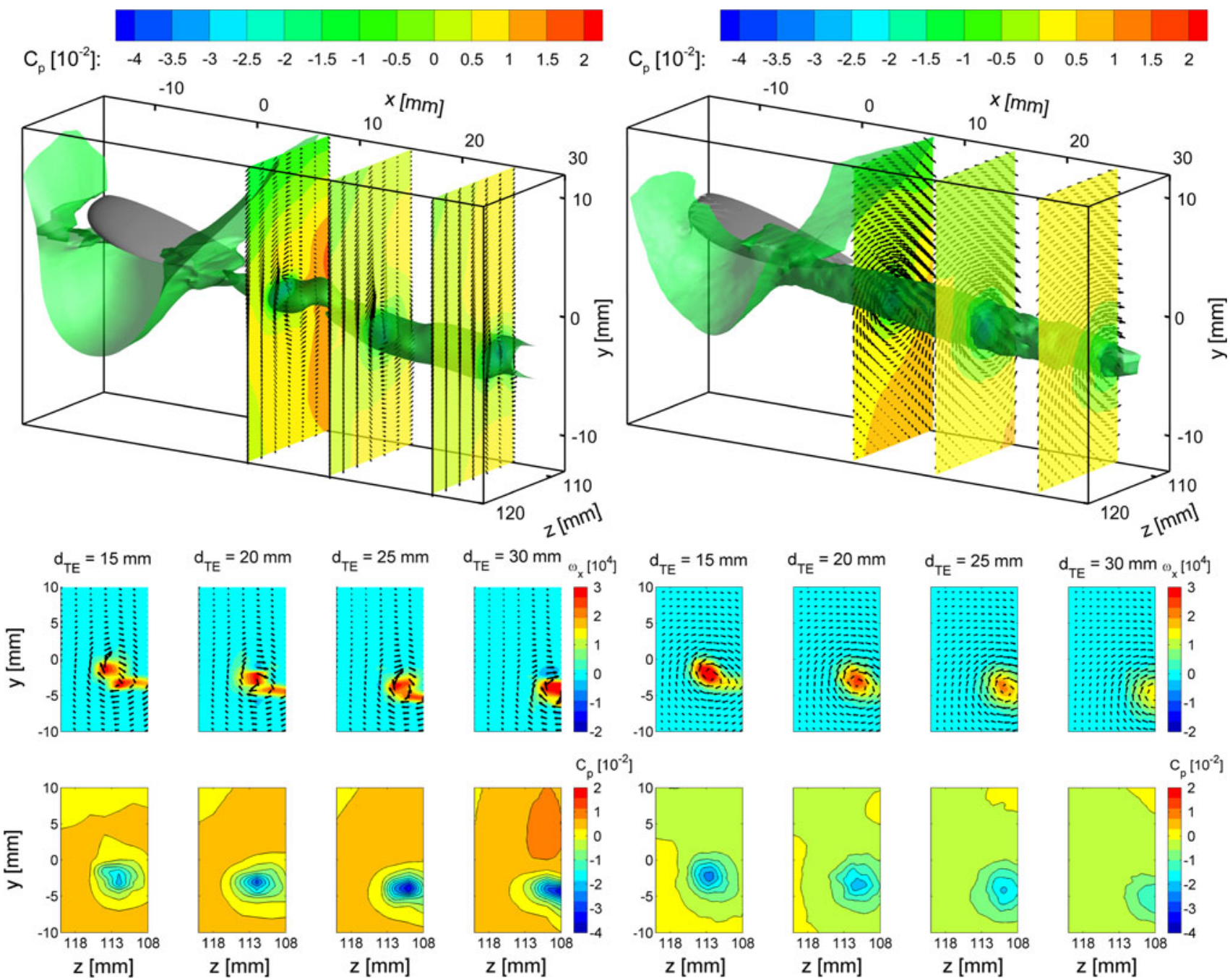

Fig. 9 Blade-tip vortex visualization, left PIV integrated pressure coefficient and $\omega_{\mathrm{x}}$ vorticity contours; right RANS computed pressure coefficient and $\omega_{\mathrm{x}}$ vorticity

vortex core have been found comparable to the ones computed from a simple analysis obtained by fitting a Lamb-Oseen laminar vortex model (Saffman et al. 1992) at the locations $d_{T E}=15 \mathrm{~mm}$ to $25 \mathrm{~mm}$, assuming at this stage a negligible helicoidal curvature of the vortex. The discrepancy between the numerical and the experimental data is attributed to the relatively limited grid resolution in the computation. Indicating the distance along the $x$ axis from the trailing edge at $r / R=92 \%$ as $d_{T E}$ (orientation shown in Fig. 9), it is estimated that the unstructured mesh ensured an average amount of 4 grid-points per $\mathrm{mm}^{2}$ in the $z-y$ planes up to $d_{T E}=15 \mathrm{~mm}$, decreasing to 2 grid-points per $\mathrm{mm}^{2}$ at $d_{T E}=30 \mathrm{~mm}$. Notwithstanding the remarkable size of the grids, both the numerical and experimental data are on the limit of their resolution to capture the inner vortex core dynamics. The 3D representation in Fig. 9 presents the vortex development at different distances from the blade trailing edge. In the limits of resolution, the iso- contours of out-of-plane vorticity $\omega_{x}$ obtained by slicing the volume at different $d_{T E}$ locations (Fig. 9) show the moderate curvature of the vortex shape, estimated to be of about $5 \mathrm{~mm}$ per $30 \mathrm{~mm}$ of elongation. The present shape is in agreement with its helicoidal motion, resulting by the combination of the blade rotational motion with the windtunnel free-stream. The imperfect misalignment of the experimental vortex distribution compared to the numerical one is within the uncertainty of the measurement. From the experimental data, the vortex peak-to-peak size is contained in a region of the $z-y$ plane of about $3 \times 3 \mathrm{~mm}^{2}$, while the numerical data confine the high vorticity in a region of about $30 \%$ higher.

\subsection{Integral loads}

The derived pressure fields together with the $3 \mathrm{D}$ velocity data are used to infer the main force components along the 

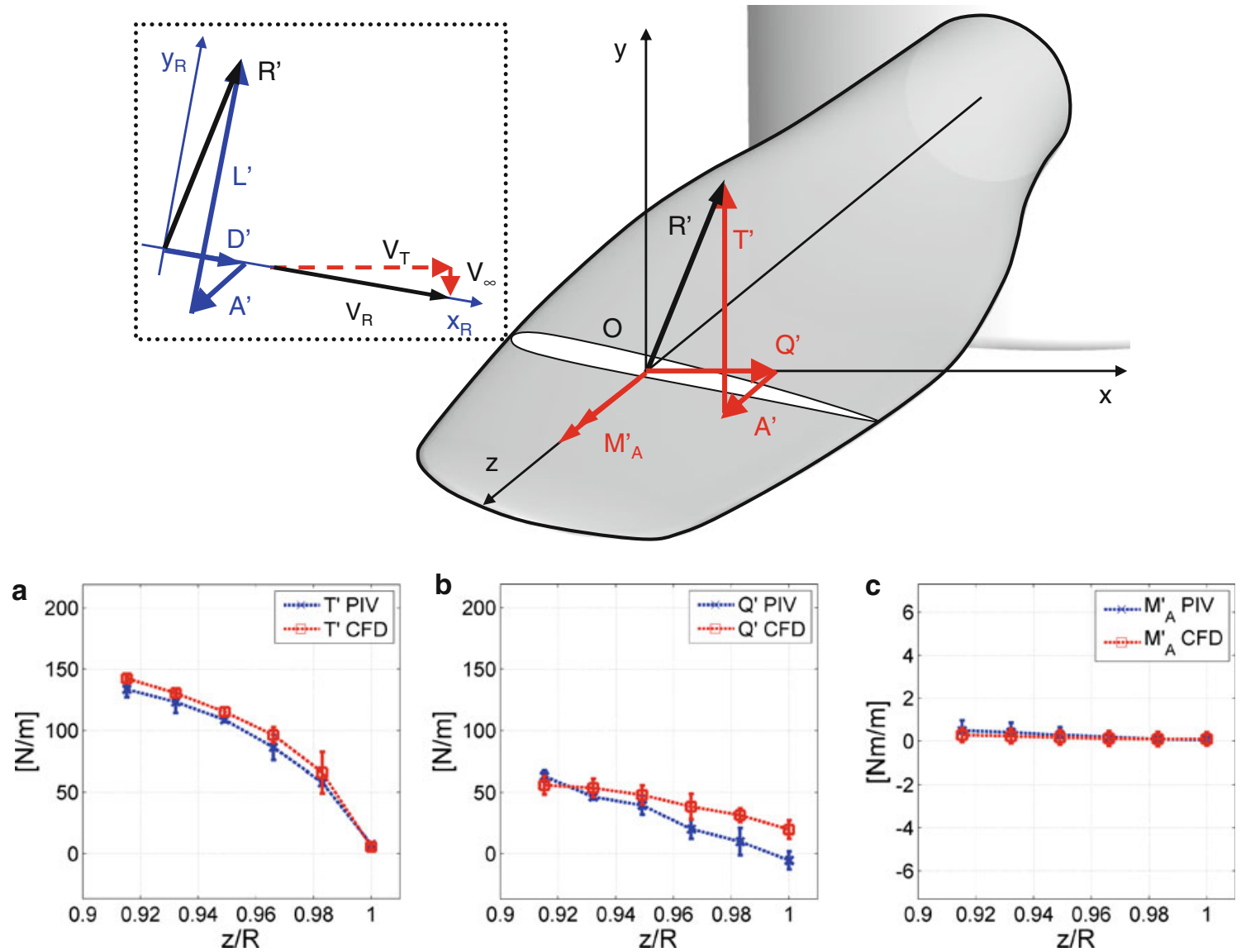

Fig. 10 Schematic of the blade load determination and cross-sectional thrust (a), torque force (b) and quarter chord torsion (c) components along the distance $z / R$

blade span by the surface-boundary contour-approach as explained in Sect. 4.2. The integration procedure, based on the one by van Oudheusden et al. 2007, has been adapted in the present investigation to retrieve the sectional forces in propeller aerodynamics. The blade itself is considered as a 3D wing, envelope of different profiles twisted along the radius, as already seen from the planar visualization in Fig. 5. Each profile contributes to the integral blade resultant load $R$ with the local lift and drag function of the location $z$. In propeller aerodynamics, the numerical and experimental cross-sectional lift, drag and pitching moment of the single blade profiles are projected onto the orthogonal $x-y-z$ frame as horizontal, tangential and torsional components, building up the blade sectional torque force $Q^{\prime}$, thrust $T^{\prime}$, and blade torsion $M_{A}^{\prime}$, in Fig. 9. This procedure for the loads estimation was also applied to the numerical flow simulation. In order to give an estimation on the uncertainty on the values of the sectional forces, the standard deviations on the different values obtained by integration over different contours were calculated and are shown as error bars for both the PIV and CFD data (Ragni et al. 2009).
The experimental cross-sectional thrust in Fig. 10a shows a decrease down to a negligible force to the tip, as already seen in the $2 \mathrm{D}$ and in the $3 \mathrm{D}$ visualization, again illustrating how the blade profile at $r / R=100 \%$ is meant to reduce the blade-tip vortex strength. The experimental results for the blade torque force, Fig. 10b, show a comparable decay toward the tip. The numerical prediction, on the other hand, due to the considerable but limited size of the mesh, displays a more diffused vorticity compared to the experimental data, which confirms the low finite drag coefficient values observed near the tip. Further analysis on those values, showed that the numerical grid resolution (mainly close to the tip where the minimum chord is identified) together with the experimental inaccuracy on the pressure values, contributes to the disagreement between the experimental and the numerical results at this particular scale, whereas the viscous and Reynolds stresses play a relatively lower role. The experimental and numerical results for the sectional torsion moment calculated at the mean-quarterchord in Fig. 10c were found to be negligible within the measurement uncertainty. 


\section{Conclusion}

The experimental investigation by stereoscopic PIV has been conducted with the intent of studying the flow on a DHC Beaver propeller running at a tip Mach number of 0.73. Planar measurements have been acquired phaselocked with the propeller motion and merged into a volume of data. Application of the PIV-based load reconstruction methodology allowed computing the 3D pressure field around the moving object. In particular, within the same experiment, the 3D velocity and pressure fields are computed and further integrated into sectional and integral loads. The experimental investigation proved that 3D features such as the acceleration field change with the blade profile, the trailing vortex dynamics, and the blade surface pressure could be captured by the nonintrusive technique, without instrumenting the propeller blade. With the drawback of a more intensive post-processing of the PIV data, the methodology retrieves further information about the blade performance by integration of the velocity and pressure data into sectional thrust and torque, in a 3D compressible test-case. Phase-locked measurements resolved the periodical flow of the blade motion, allowing using the blade-based frame of reference to reduce the amount of data collected and to simplify the pressure computation. Integration of the governing equations in the compressible regime was used as a direct comparison of the pressure field coefficients derived from the measured velocity data, with the ones of a numerical RANS-periodical steady computation of the entire propeller model. A quantitative analysis of the pressure fields of the blade demonstrated how the propeller blade becomes less tractive as the measurement planes move to the tip, determining a corresponding decrease in the blade torque force due to the weakness of the trailing vortex. The numerical behavior confirmed the pressure analysis, providing comparable results with maximum differences of the order of $10 \%$, ascribed to uncertainties in the representation of the real blade shape in the numerical model. Further integration of the velocity and pressure fields by means of a momentum-integral approach allowed determining the distribution of the load on the blade itself. To a quantitative comparison, the sectional PIV computed thrust has been found the most in agreement with the one from the simulation data, showing the expected decrease in traction up to the tip in both the CFD and PIV curves. The experimental sectional torque force, due to the more localized extension of the blade wake and to its lower impact, compares favorably to the numerical data mainly at the inboard part of the measurement domain, while a consistent deviation between experiment and simulation was observed at the immediate tip region.
Acknowledgments This work is supported by the Dutch Technology Foundation STW (grant n. 07645).

Open Access This article is distributed under the terms of the Creative Commons Attribution Noncommercial License which permits any noncommercial use, distribution, and reproduction in any medium, provided the original author(s) and source are credited.

\section{References}

Anderson JD (1991) Fundamentals of aerodynamics, 2nd edn. McGraw Hill, New York

Berton E, Maresca C, Favier D (2004) A new experimental method for determining local airloads on rotor blades in forward flight. Exp Fluids 37:455-457

Bousquet JM, Gardarein P (2003) Improvements on computations of high speed propeller unsteady aerodynamics. Aerosp Sci Technol 7(6):465-472

Boyle FJ, Flaherty MPO, Eaton JA (1999) Comparison of PIV measurements with CFD predictions for a high speed propeller. 35th AIAA/ASME/SAE/ASEE joint propulsion conference and exhibit. Los Angeles, California, AIAA 99-2387

Cheng HK, Chow R, Melnik F (1981) Lifting-line theory of swept wings based on the full potential theory. J Appl Math Phys 32(5):481-496

Cook LP, Cole JD (1978) Lifting-line theory for transonic flow. SIAM J Appl Math 35(2):209-228

Datta A, Nixon M, Chopra I (2007) Review of rotor loads prediction with the emergence of rotorcraft CFD. J Am Helicopter Soc 52(4):287-317

Elsinga GE, van Oudheusden BW, Scarano F (2005) Evaluation of aero-optical distortion effects in PIV. Exp Fluids 39(2):245-256

Favier D, Maresca C, Ettaouil A (1989) Numerical investigation of isolated propeller wakes in axial flight. J Aircr 26:837-846

Kushan MC, Diltemiz SF, Sackesen İ (2007) Failure analysis of an aircraft propeller. Eng Fail Anal 14:1693-1700

Lee HC, Hwang YH, Kim TG (2004) Failure of aircraft propeller assembly. Eng Fail Anal 11:305-312

Ludford GSS (1951) The behavior at infinity of the potential function of a two-dimensional subsonic compressible flow. J Math Phys 25:117-130

Noca F, Shiels D, Jeon D (1999) A comparison of methods for evaluating time-dependent fluid-dynamic forces on bodies, using only velocity fields and their derivatives. J Fluid Struct 13:551-578

Ragni D, Ahok A, van Oudheusden BW, Scarano F (2009) Surface pressure and aerodynamic loads determination of a transonic airfoil based on particle image velocimetry. Meas Sci Tech 20(7): $1-14$

Ragni D, van Oudheusden BW, Scarano F (2010) Drag coefficient accuracy improvement by means of particle image velocimetry for a transonic NACA0012 airfoil. Meas Sci Tech 22:1

Ragni D, van Oudheusden BW, Scarano F (2011) Non-intrusive aerodynamic loads analysis of an aircraft propeller blade. Exp Fluids doi:10.1007/s00348-011-1057-7

Ramasamy M, Leishman JG (2006) Benchmarking PIV with LDV for Rotor Wake Vortex Flows, AIAA 2006-3479, 24th Applied Aerodynamics Conference 5-8 June 2006. San Francisco, California

Roosenboom EWM, Stürmer A, Schröder A (2010) Advanced experimental and numerical validation and analysis of propeller slipstream flows. AIAA 47(1):284-291 
Saffman PG, Ablowitz MJ, Hinch JE, Ockendon JR, Olver PJ (1992) Vortex dynamics. Cambridge University Press, Cambridge

Scarano F, Riethmuller ML (2000) Advances in iterative multi-grid PIV image processing. Exp Fluids 29(1):51-60

Schrijer FFJ, Scarano F (2007) Particle slip compensation in steady compressible flows, $7^{\text {th }}$ Int. Symp. on Particle Image Velocimetry. Rome, Italy

Schrijer FFJ, Scarano F (2008) Effect of predictor-corrector filtering on the stability and spatial resolution of iterative PIV interrogation. Exp Fluids 45(5):927-941

Stepnov MN, Seregin AS, Leonova OV, Sukhorosov YL, Kulikov EI, Dunaev ÉV (1977) Fatigue resistance of aircraft propeller blades. Strength Mater 9(5):550-554

Trefethen L (2000) Spectral methods in Matlab. SIAM, Philadelphia
Unal MF, Lin JC, Rockwell D (1997) Force prediction by PIV imaging: a momentum-based approach. J Fluids Struct 11:965-971

van Oudheusden BW, Scarano F, Casimiri EWM (2006) Nonintrusive load characterization of an airfoil using PIV. Exp Fluids 40(6):988-992

van Oudheusden BW, Scarano F, Roosenboom EWM, Casimiri EWF, Souverein LJ (2007) Evaluation of integral forces and pressure fields from planar velocimetry data for incompressible and compressible flows. Exp Fluids 43(2-3):153-162

Westerweel J (1993) Digital particle image velocimetry. Delft University Press, Delft

Wieneke B (2005) Stereo-PIV using self-calibration on particle images. Exp Fluids 39:267-280 